\section{Homicidios en Medellín, Colombia, entre 1990 y 2002: actores, móviles y circunstancias}

\author{
Homicides in Medellín, Colombia, from 1990 \\ to 2002: victims, motives and circumstances
}

\author{
Marleny Cardona 1,2 \\ Héctor Iván García 1,3 \\ Carlos Alberto Giraldo 1,3 \\ María Victoria López 1,4 \\ Clara Mercedes Suárez 1,5 \\ Diana Carolina Corcho 1,6 \\ Carlos Hernán Posada 1,3 \\ María Nubia Flórez 6
}

\begin{abstract}
1 Grupo de Investigación en Violencia Urbana, Universidad de Antioquia, Medellin, Colombia.

2 Departamento de Economía, Universidad EAFIT, Medellín, Colombia.

3 Facultad de Medicina, Universidad de Antioquia, Medellín, Colombia.

4 Facultad de Enfermería, Universidad de Antioquia Medellín, Colombia. 5 Maestría en Salud Pública, Universidad de Antioquia, Medellín, Colombia. 6 Instituto Nacional de Medicina Legal y Ciencias Forenses, Regional Nor-Occidente, Medellín, Colombia.

Correspondencia H. I. García Facultad de Medicina Universidad de Antioquia. Carrera 51D \# 62-29, Medellín, Colombia. higarcia@quimbaya.udea.edu.co

Abstract

In Medellin, Colombia, homicide has been the first cause of morbidity and mortality for 20 years. Medellin has the highest homicide rates of all major cities in Latin America. This study describes the victims, motives, and circumstances in homicides in Medellín from 1990 to 2002. The period included 55,365 homicides, of which 1,394 were randomly studied. Of this sample, 93.6\% (95\%CI: 92.2\%-94.8\%) were males, 77.0\% (95\%CI: 75.0\%-79.5\%) less than 35 years of age, onefourth had consumed alcohol, and nine out of ten were killed with firearms. The main motives were revenge and armed robbery. $37.0 \%$ (95\%CI: $34.0 \%-41.0 \%)$ of the victims lived in the lowest socioeconomic stratum of the city. Characteristics of homicides in Medellin have remained unchanged since the 1980s, when the most violent period in the city's history began. The most heavily affected groups are young males who live and die in poor neighborhoods, and the murders are individual acts that leave no wounded behind.
\end{abstract} cagiraldo@epm.net.co
Violence; Homicide; Socioeconomic Factors

\section{Introducción}

La violencia es una forma extrema de resolución de los conflictos sociales entre las personas y los colectivos. Genéricamente ha sido caracterizada como la imposición de la fuerza al servicio de determinados intereses, ejercida en condiciones de asimetría, con una direccionalidad específica, que resulta en la limitación o la negación de la víctima 1 . El homicidio, en particular, es la forma suprema de expresión de la violencia en cuanto priva a la víctima de la totalidad de sus derechos y en tanto tiene un profundo significado social.

Medellín está ubicada al noroccidente de Colombia y es considerada la segunda ciudad del país por su desarrollo industrial y por su población. En 1990 tenía 1.641.341 habitantes y 2.025.293 en el 2002. En ella, el homicidio y otras formas de violencia públicas se reconocen como el principal problema social, económico, de salud pública y de seguridad ciudadana, que se ha generalizado a todos los ámbitos de la cotidianidad social, que afecta la calidad de vida de sus habitantes y que no respeta ningún tipo de instituciones, educativas o de salud 2 .

En la ciudad, el homicidio es la primera causa de mortalidad general desde 1986 y su participación en el total de muertes se incrementó del 3,5\% en 1976 , al $8,0 \%$ en 1980 , al 17,0\% en 1985 y alcanzó el máximo del 42,0\% en 1991; año a partir del cual descendió hasta 
el 30,0\% en 1995 y 28,0\% en 2002 3,4,5,6. Entre 1990 y 1999 hubo 45.434 homicidios y en los tres años del segundo milenio se dieron 9.931 muertes (promedio anual 3.310). La tasa anual de homicidio en el período (Figura 1) descendió paulatinamente desde cifras superiores a 320 por cien mil habitantes en los primeros años, hasta tasas que rodearon los 160 por cien mil habitantes en los últimos 7. No obstante, las menores tasas de Medellín fueron más elevadas que las mayores tasas de grandes ciudades del continente americano, como Río de Janeiro con 61 por cien mil habitantes en 1992 y São Paulo con 44 por cien mil habitantes en 1994 y 2001 8,9 (Guerrero R. Comunicación personal; 1996) y que las de grandes capitales de departamento del país, como Bogotá con 80 por cien mil habitantes en 1993, Barranquilla con 49 por cien mil habitantes en 1996, Cúcuta con 105 por cien mil habitantes en 2000 y Cali con 124 por cien mil habitantes en 1994 10,11.

Un estudio previo sobre la violencia homicida en Medellín permitió establecer, como características relevantes, el que las víctimas eran hombres jóvenes entre 15 y 34 años de edad, que en el 36,0\% de los casos estaban bajo efectos del alcohol en el momento de la muerte. Cuando se logró establecer el móvil (40,0\% de los casos) hubo un predominio de los ajustes de cuentas, de las riñas y de los atracos. Hay ma- yor riesgo de que los hechos que conducen al homicidio ocurran en lugares ubicados en los estratos bajos y que las víctimas procedan de esos mismos estratos. Asimismo, es más frecuente que los homicidios se presenten por las noches, especialmente durante los fines de semana y que la calle sea el lugar donde hay una mayor ocurrencia 12 .

A pesar de la magnitud del problema, de sus efectos en el conjunto de la sociedad y de la convicción cada vez más compartida de que la violencia ha puesto en entredicho la viabilidad del desarrollo de la ciudad, Medellín no tiene un sistema unificado de información de homicidios y otras formas de violencia de alcance poblacional que permita conocer sus dinámicas y orientar la toma de decisiones para la búsqueda de soluciones. El conocimiento del que se dispone proviene de informes de investigaciones que se ocupan de dilucidar aspectos parciales y de las cifras fragmentarias recopiladas por algunas autoridades locales.

En consecuencia, es aplicable a Medellín lo que expresa Rubio (Comunicación personal; 1998) con respecto a Latinoamérica: "en abierto contraste con esta percepción acerca de la gravedad e importancia de la violencia son irrisorios los esfuerzos que se están haciendo en la actualidad para tratar de precisar la naturaleza y medir la magnitud, de uno de los principales

Figura 1

Evolución de la tasa de mortalidad por homicidios en Colombia y Medellín, 1975-2002.

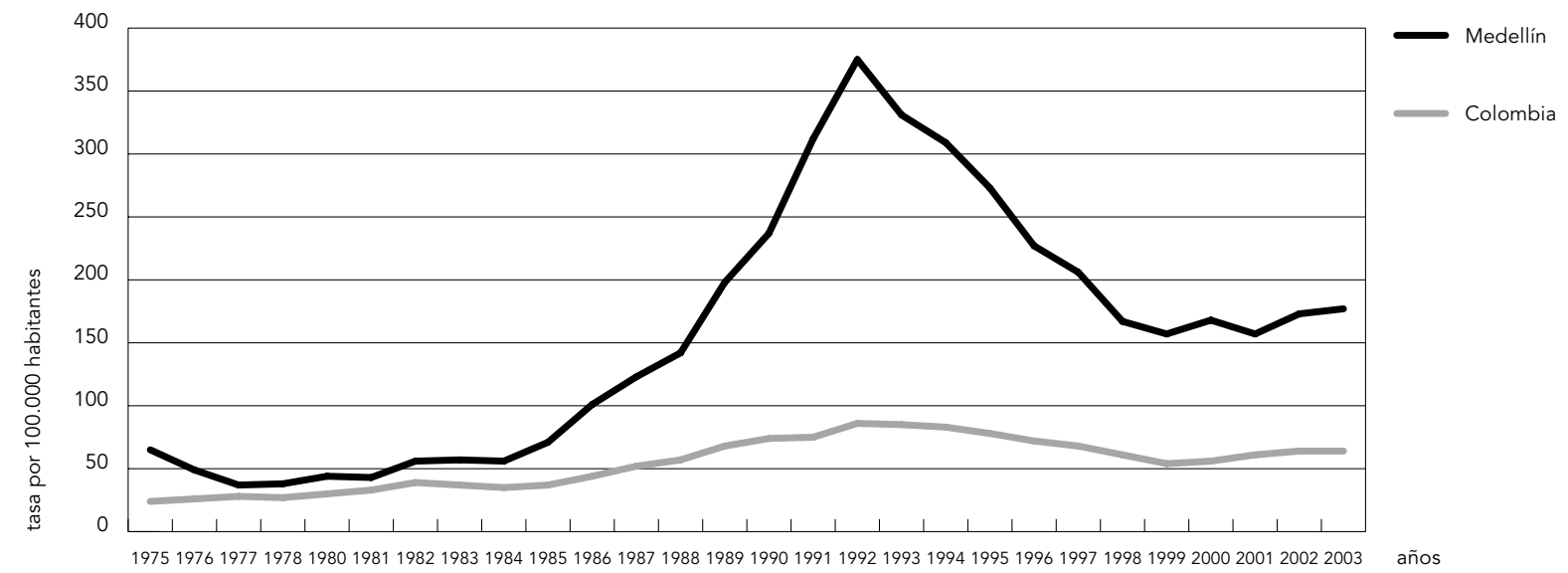

Fuente: Instituto Nacional de Medicina Legal y Ciencias Forenses, Regional Noroccidente. Boletín de Prensa; 2002. 
problemas que, en eso parece haber consenso, está agobiando a la región latinoamericana. Lo que se gasta actualmente en medir los precios, los medios de pago, las cuentas fiscales, la balanza de pagos, el empleo o las cuentas nacionales no guarda proporción, dada la trascendencia que le asignan los ciudadanos a los distintos problemas, con lo que se está gastando en medir el crimen y la violencia".

De acuerdo con las consideraciones anteriores, esta investigación buscó identificar las características de los actores, las circunstancias y los móviles de los hechos violentos que tuvieron víctimas fatales en Medellín en los últimos doce años, 1990/2002; dándole continuidad a un estudio de características similares sobre la década de los años ochenta 12 y con ello obtener una visión panorámica de esta epidemia de homicidios ocurrida en la ciudad en los últimos veinte años. Mediante el estudio de la violencia en un período de tiempo más largo del que convencionalmente se analiza en nuestro medio, se busca abrir posibilidades de intervención y buscar soluciones, aportando argumentos que sirvan para la formulación y ejecución de políticas y de programas de intervención.

\section{Métodos}

Se realizó un estudio descriptivo, retrospectivo, longitudinal. La población de referencia fueron los 55.365 homicidios que ocurrieron en Medellín entre 1990 y 2002 y a los que se les practicó necropsia en el Instituto Nacional de Medicina Legal y Ciencias Forenses, Regional Noroccidente (INMLyCF). Se excluyeron los registros de los homicidios ocurridos en el área rural y en otros municipios y aquellos en los que no se logró esclarecer si se trataba de un homicidio.

El muestreo fue aleatorio, estratificado para los 13 años del período, con el fin de garantizar participación de los años con menor número de homicidios. El tamaño de la muestra de cada estrato fue proporcional al número de muertes ocurridas en él. La selección final de las unidades de análisis, dentro de cada estrato, se hizo en forma sistemática. El cálculo del tamaño de la muestra se hizo con base en los siguientes parámetros: proporción a estimar del conocimiento del móvil del homicidio en cada período del 35,0\% (con base en el estudio de García \& Vélez 12 ), nivel de confianza del $95 \%$ y un error de muestreo de 2,5\%. El total calculado fue de 1.364 unidades de análisis y se adicionaron treinta unidades más, tomando finalmente datos de 1.394 homicidios.
Las fuentes de los datos fueron el Acta de levantamiento del cadáver que diligencian los funcionarios de la Fiscalía General de la Nación, el informe de la necropsia practicada por el médico forense y la hoja de ruta elaborada en el INMLyCF.

El formulario para la recolección de datos incluyó variables relacionadas con la víctima, su procedencia, las circunstancias temporales, geográficas y descriptivas del hecho que ocasionaron el homicidio, y los móviles consignados por los funcionarios que hicieron el levantamiento del cadáver. Este formulario se probó con 50 unidades de análisis distribuidas en los 13 años y seleccionadas de forma aleatoria en el archivo del INMLyCF.

La recolección de los datos la hicieron tres de los investigadores (C. M. S., D. C. C. y C. H. P.) entrenados para reducir al máximo los sesgos. El control de calidad de la información se hizo por dos de los investigadores (C. M. S. y H. I. G.), de forma continua durante el período de recolección de la información, con base en la consistencia y coherencia interna. La sistematización de los datos se hizo en los programas Microsoft Excel ${ }^{\circledR} 2002$ y Epi Info versión 2002 y el análisis estadístico en este y en SPSS ${ }^{\circledR}$ 11.5.

La descripción de los actores, las circunstancias y los móviles de los homicidios se hizo con porcentajes para las variables categóricas y se usaron promedios, desviaciones estándar y rangos para las variables numéricas. La cantidad de alcohol ingerida por la víctima fue medida según los miligramos de etanol por cien miligramos de sangre total y se clasificó en grados de embriaguez así: primer grado entre 40 y 99 miligramos por ciento; segundo grado entre 100 y $149 \mathrm{mgs} \%$ y tercer grado igual o mayor a $150 \mathrm{mgs} \%$ 13. Para la ubicación geográfica de los barrios, comunas y zonas del lugar de residencia del muerto y de los hechos del homicidio, así como las relaciones de vecindad entre ellos, se utilizaron los mapas político-administrativos proporcionados por el Departamento Administrativo de Planeación Metropolitana, adscrito al municipio de Medellín. Se usó la estratificación socioeconómica definida por las Empresas Públicas de Medellín (entidad que provee los servicios públicos domiciliarios) para cada uno de los barrios, en los diferentes años del período.

Para medir el riesgo de morir por homicidio según el barrio y la comuna de residencia y de ocurrencia del homicidio, se diseñaron los indicadores Índice H/P Barrio de Residencia, Índice H/P Comuna de Residencia, Razón H/P Barrio del Hecho, Razón H/P Comuna del Hecho, los cuales se calcularon así: 
- Índice H/P Barrio Residencia = $\%$ de homicidios de residentes en el barrio $\%$ población residente en el barrio a mitad del período

- Índice H/P Comuna Residencia = $\%$ de homicidios de residentes en la comuna \% población residente en la comuna a mitad del período

- Razón H/P Barrio Hecho = $\%$ homicidios ocurridos en el barrio \% población del barrio a mitad del período

- Razón H/P Comuna Hecho = $\%$ homicidios ocurridos en la comuna $\%$ población de la comuna a mitad del período

Estos indicadores permiten hacer comparaciones entre los espacios geográficos, al poner en relación los homicidios por la población existente en ellos. Si la muerte por homicidio se presentara por azar en un espacio geográfico de residencia o de muerte, independiente de los contextos y eventos sociales que en él se dan, el indicador debería ser igual o cercano a 1,0 . Sus valores fluctúan entre 0,0 , en los casos en que no hubo muertes de residentes en el lugar o no hubo homicidios en él, y valores mayores de 1,0 para los espacios donde hay riesgo de morir por pertenecer a él o por estar en él en un momento dado. El riesgo se clasificó en $\mathrm{Ba}$ jo cuando el Índice tuvo un valor inferior a 0,9 ; Medio cuando el Índice estuvo entre 0,91 y 2,0, y Alto cuando el Índice fue superior a 2,0.

Para valorar el riesgo de morir que tiene una persona según el estrato socioeconómico (denominados Bajo-bajo, Bajo, Medio-bajo, Medio-medio, Medio-alto y Alto) al cual pertenece su residencia, se definió el Índice H/P Estrato de Residencia calculado como:

- Índice H/P Estrato de Residencia = $\%$ de homicidios de residentes en el estrato \% población residente en el estrato a mitad del período

\section{Resultados}

En Medellín, entre los años 1990 y 1999 hubo 45.434 homicidios y 9.931 de 2000 a 2002, para un total de 55.365 en los 13 años. El menor número de homicidios se presentó en 1998 con 2.854 y el año con mayor número fue 1991 con 6.658. En la Figura 1 se muestra la tendencia de la tasa de homicidio por cien mil habitantes en la ciudad durante el período de estudio.

Las características demográficas, las circunstancias y los móviles de los homicidios se presentan en la Tabla 1. En el total del período predominaron los hombres (93,6\%; IC95\%: 92,2\%94,8\%), con variaciones entre el 88,0\% (IC95\%: 82,0\%-93,0\%) en 2002 y el 98,0\% (IC95\%: 92,0\%99,7\%) en 1998. El 77,4\% (IC95\%: 75,0\%-79,5\%) de las muertes ocurrieron en menores de 35 años, el 47,4\% (IC95\%: 45,0\%-50,0\%) tenía entre 15 y 24 años y el 28,3\% (IC95\%: 26,0\%-31,0\%) tenían entre 25 y 34 años.

De los 1.394 casos de la muestra, se hizo determinación de alcoholemia a 1.304 cadáveres lo que corresponde al $94,0 \%$. De ellos, el $24,1 \%$ (IC95\%: 22,0\%-26,5\%) tenía algún nivel positivo de alcoholemia. La positividad del alcohol en la sangre fluctuó entre 13,6\% (IC95\%: 7,0\%$22,6 \%$ ) en el año 2000 y 38,1\% (IC95\%: 29,0\%$47,5 \%$ ) en 1990. Tres de los 10 sujetos a quienes se les practicaron exámenes para otras sustancias psicoactivas resultaron positivos para cocaína; el otro lo fue para benzodiazepinas.

En cuanto a los métodos utilizados se encontró que aproximadamente 9 de cada 10 homicidios se realizaron con armas de fuego en todo el período. Se registró una mayor variación en los años 1994 y 1995 en los que las armas de fuego se utilizaron en un 84,0\% (IC95\%: 75,0\%-91,0\%) de los casos mientras que las armas cortopunzantes se utilizaron en un $14,8 \%$ (IC95\%: $8,0 \%-24,0 \%$ ) y $14,2 \%$ (IC95\%: $8,0 \%$ $22,0 \%)$ respectivamente. Los artefactos explosivos, como bombas, tuvieron una participación escasa en el período con menos de un evento por año, aunque murieron 6 personas en promedio en cada uno de ellos, mientras con el arma de fuego el promedio fue 1,3.

En los registros de Medicina Legal, el móvil del homicidio estaba consignado por los funcionarios de la Fiscalía en 215 casos $(15,4 \%$; IC95\%: 13,6\%-17,5\%). El conocimiento del móvil varió entre el 29,1\% (IC95\%: 19,0\%-40,0\%) en 1997 y el 6,4\% (IC95\%: 2,8\%-12,0\%) en 1990; en los casos en los cuales estaba consignado, predominaron el ajuste de cuentas (7,0\%; IC95\%: $5,7 \%-8,5 \%)$ y el atraco (4,5\%; IC95\%: $3,5 \%-5,8 \%$ ). En todos los móviles fue más frecuente el uso de las armas de fuego (mayor del $87,0 \%$ ), excepto en las riñas donde el arma cortopunzante se usó en el $42,0 \%$.

La relación del muerto con el homicida durante el hecho violento se identificó en 38,4\% (IC95\%: 35,3\%-41,8\%) de los casos; 23 de ellos eran agresores (1,6\%; IC95\%: 1,1\%-2,5\%), de los cuales 11 (47,8\%; IC95\%: 27,0\%-69,0\%) ocurrieron en atracos y 8 (34,8\%; IC95\%: $13,0 \%-75,0 \%)$ 
Actores, circunstancias y móviles de los homicidios. Medellín, Colombia, 1990/2002.

\begin{tabular}{|c|c|c|}
\hline Característica & $\mathrm{n}$ & Porcentaje (IC95\%) \\
\hline Sexo masculino & 1.305 & $93,6(92,2-94,8)$ \\
\hline Razón hombre/mujer en el período & \multicolumn{2}{|c|}{$15: 1$} \\
\hline Mínimo: año 2002 & \multicolumn{2}{|c|}{$7: 1$} \\
\hline Máximo: año 1998 & \multicolumn{2}{|c|}{$44: 1$} \\
\hline Edad en años (promedio y desviación estándar) & \multicolumn{2}{|c|}{$27,6(10,7)$} \\
\hline \multicolumn{3}{|l|}{ Grupos de edad (en años) } \\
\hline $0-14$ & 24 & $1,7(1,1-2,6)$ \\
\hline $15-19$ & 309 & $22,3(20,1-24,6)$ \\
\hline $20-24$ & 348 & $25,1(22,8-27,5)$ \\
\hline $25-29$ & 230 & $16,6(14,7-18,7)$ \\
\hline $30-34$ & 162 & $11,7(10,1-13,5)$ \\
\hline $35-39$ & 128 & $9,2(7,8-10,9)$ \\
\hline $40-80$ & 186 & $13,4(10,4-17,2)$ \\
\hline \multicolumn{3}{|l|}{ Grado de embriaguez $(n=1.304)$} \\
\hline Negativo & 990 & $75,9(73,5-78,2)$ \\
\hline Primero (40-99mg\%) & 101 & $7,7(6,4-9,4)$ \\
\hline Segundo (100-149mg\%) & 107 & $8,2(6,8-9,9)$ \\
\hline Tercero (150 y más mg\%) & 106 & $8,1(6,7-9,8)$ \\
\hline \multicolumn{3}{|l|}{ Posible móvil } \\
\hline Ajuste de cuentas & 97 & $7,0(5,7-8,5)$ \\
\hline Atraco & 63 & $4,5(3,5-5,8)$ \\
\hline Riña & 19 & $1,4(0,8-2,2)$ \\
\hline Enfrentamiento armado & 17 & $1,2(0,7-2,0)$ \\
\hline Limpieza social & 14 & $1,0(0,6-1,7)$ \\
\hline Intervención legal & 4 & $0,3(0,1-1,8)$ \\
\hline Maltrato conyugal & 1 & $0,1(0,0-0,5)$ \\
\hline Desconocido & 1.179 & $84,6(82,5-86,4)$ \\
\hline \multicolumn{3}{|l|}{ Estrato socioeconómico de residencia del muerto } \\
\hline Bajo bajo y Bajo & 519 & $37,3(34,0-41,0)$ \\
\hline Medio bajo y Medio & 481 & $34,5(31,0-39,0)$ \\
\hline Medio alto y Alto & 26 & $1,9(1,1-3,2)$ \\
\hline Fuera de Medellín & 48 & $3,5(2,5-5,0)$ \\
\hline Miserable (residía en la calle) & 10 & $0,7(0,4-1,4)$ \\
\hline Desconocido & 310 & $22,2(20,0-25,0)$ \\
\hline \multicolumn{3}{|l|}{ Estrato socioeconómico del lugar del hecho } \\
\hline Bajo-bajo y Bajo & 551 & $39,6(36,0-44,0)$ \\
\hline Medio-bajo y Medio & 644 & $46,2(42,0-51,0)$ \\
\hline Medio-alto y Alto & 62 & $4,5(3,0-6,0)$ \\
\hline Rural & 59 & $4,2(3,0-6,0)$ \\
\hline Desconocido & 78 & $5,6(5,0-7,0)$ \\
\hline \multicolumn{3}{|l|}{ Tipo de arma } \\
\hline De fuego & 1.233 & $88,6(87,0-90,0)$ \\
\hline Cortopunzante & 133 & $9,6(8,0-11,0)$ \\
\hline Explosivos & 9 & $0,6(0,3-1,3)$ \\
\hline Otras (estrangulamiento, contundente, sumergimiento) & 17 & $1,2(0,5-2,9)$ \\
\hline \multicolumn{3}{|l|}{ Tipo de actor } \\
\hline Agredido & 512 & $36,7(34,2-39,3)$ \\
\hline Agresor & 23 & $1,6(1,1-2,5)$ \\
\hline Desconocido & 859 & $61,6(59,0-64,2)$ \\
\hline
\end{tabular}


en enfrentamientos armados o intervenciones legales de los organismos militares y de policía del Estado.

En 89,7\% (IC95\%: 85,0\%-95,0\%) de los hechos en los que hubo homicidios tuvieron como resultado una víctima única. En 93,0\% (IC95\%: 88,0\%-98,0\%) de esos incidentes no hubo otras personas heridas. Esto fue similar en todos los años del estudio, excepto en 1995 cuando los hechos con muerto único y sin heridos fueron del $79,5 \%$ y $77,1 \%$, en 1998 que fueron $87,5 \%$ y $78,1 \%$, y en 1996 cuando fueron $76,9 \%$ y $73,7 \%$ respectivamente.

En la Tabla 2 se presentan algunas de las características que tuvieron los móviles de los homicidios que fueron identificados por los funcionarios de la Fiscalía en el momento del levantamiento del cadáver. Todas estas características tuvieron diferencias estadísticas significativas $(p<0,05)$ entre los móviles identificados.

La mayoría de homicidios sucedieron por la noche (56,6\%; IC95\%: 54,0\%-59,0\%), especial- mente entre las 18:00 y las 0:00 (38,0\%; IC95\%: $31,0 \%-47,0 \%)$. La proporción de muertes fue mayor de lunes a viernes (63,2\%; IC95\%: 59,0\%$67,0 \%$ ), pero si se relativiza este valor por el porcentaje de horas existentes en el período, el riesgo de morir durante el fin de la semana (60 horas existentes entre las 18:00 del viernes y las 6:00 del lunes) es casi dos veces al riesgo del resto de la semana $(1,3$ contra 0,8$)$. La distribución de muertes según meses y trimestres del año no tuvieron diferencias.

El 95,6\% (IC95\%: 94,0\%-97,0\%) de las personas asesinadas en el período residían en la ciudad y más de la mitad $(56,3 \%)$ en las zonas NorOriental (34,4\%; IC95\%: 32,0\%-37,0\%) y NorOccidental (21,9\%; IC95\%: 20,0\%-25,0\%), mientras que en la Sur-Oriental lo hacían el 0,8\% (IC95\%: 0,4\%-1,6\%). El 0,9\% (IC95\%: 0,5\%-1,7\%) de los muertos vivían en la calle. Por comunas (agrupaciones administrativas de los barrios), las localizadas en el norte de la ciudad fueron las que aportaron más muertes: Popular $(9,2 \%$;

Características y relaciones geográficas de los móviles en los homicidios ocurridos en Medellín, Colombia, $1990 / 2002$.

\begin{tabular}{|c|c|c|c|c|c|c|c|c|c|c|}
\hline \multirow{2}{*}{$\begin{array}{l}\text { Posible } \\
\text { Móvil }\end{array}$} & \multirow[t]{2}{*}{ Edad* $^{*}$} & \multirow{2}{*}{$\begin{array}{l}\text { Arma de } \\
\text { fuego } \\
\%(I C 95 \%)\end{array}$} & \multirow{2}{*}{$\begin{array}{c}\text { Alcoholemia } \\
\text { negativa } \\
\%(I C 95 \%)\end{array}$} & \multirow{2}{*}{$\begin{array}{l}\text { Homicidio } \\
\text { individual** } \\
\%(I C 95 \%)\end{array}$} & \multirow{2}{*}{$\begin{array}{l}\text { Muerto } \\
\text { agresor } \\
\% \text { (IC95\%) }\end{array}$} & \multirow{2}{*}{$\begin{array}{l}\text { Hubo algún } \\
\text { herido } \\
\% \text { (IC95\%) }\end{array}$} & \multicolumn{4}{|c|}{ Relación barrio de residencia y hecho } \\
\hline & & & & & & & $\begin{array}{c}\text { Igual } \\
\text { barrio } \\
\%(I C 95 \%)\end{array}$ & $\begin{array}{c}\text { Barrio } \\
\text { limítrofe } \\
\% \text { (IC95\%) }\end{array}$ & $\begin{array}{c}\text { Barrio } \\
\text { alejado } \\
\%(I C 95 \%)\end{array}$ & $\begin{array}{l}\text { Residente } \\
\text { en la calle } \\
\% \text { (IC95\%) }\end{array}$ \\
\hline $\begin{array}{l}\text { Ajuste de } \\
\text { cuentas } \\
(n=97)\end{array}$ & $25(10)$ & $\begin{array}{c}93,8 \\
(87,0-98,0)\end{array}$ & $\begin{array}{c}81,5 \\
(72,0-89,0)\end{array}$ & $\begin{array}{c}85,2 \\
(76,0-92,0)\end{array}$ & $\begin{array}{c}1,2 \\
(0,0-6,6)\end{array}$ & $\begin{array}{c}16,1 \\
(5,0-41,0)\end{array}$ & $\begin{array}{c}48,2 \\
(37,0-59,0)\end{array}$ & $\begin{array}{c}12 \\
(6,0-21,0)\end{array}$ & $\begin{array}{c}40 \\
(29,0-51,0)\end{array}$ & 0 \\
\hline $\begin{array}{l}\text { Atraco } \\
(n=63)\end{array}$ & $34(13)$ & $\begin{array}{c}87,3 \\
(77,0-94,0)\end{array}$ & $\begin{array}{c}81,7 \\
(70,0-91,0)\end{array}$ & $\begin{array}{c}90,6 \\
(79,0-97,0)\end{array}$ & $\begin{array}{c}19,6 \\
(10,0-32,0)\end{array}$ & $\begin{array}{c}13,5 \\
(3,0-34,0)\end{array}$ & $\begin{array}{c}23,0 \\
(12,0-38,0)\end{array}$ & $\begin{array}{c}19 \\
(9,0-33,0)\end{array}$ & $\begin{array}{c}55 \\
(40,0-70,0)\end{array}$ & $(0,1-11,0)$ \\
\hline $\begin{array}{l}\text { Riña } \\
(n=19)\end{array}$ & 34 (13) & $\begin{array}{c}47,4 \\
(24,0-71,0)\end{array}$ & $\begin{array}{c}42,1 \\
(20,0-67,0)\end{array}$ & $\begin{array}{c}93,8 \\
(70,0-99,8)\end{array}$ & 0,0 & $\begin{array}{c}15,4 \\
(2,0-45,0)\end{array}$ & $\begin{array}{c}8,0 \\
(0,2-39,0)\end{array}$ & $\begin{array}{c}17 \\
(2,0-48,0)\end{array}$ & $\begin{array}{c}75 \\
(43,0-95,0)\end{array}$ & - \\
\hline $\begin{array}{l}\text { Enfrentami- } \\
\text { ento armado } \\
(n=17)\end{array}$ & $23(11)$ & 100,0 & $\begin{array}{c}81,2 \\
(54,0-96,0)\end{array}$ & $\begin{array}{c}30,8 \\
(9,0-61,0)\end{array}$ & $\begin{array}{c}63,6 \\
(31,0-89,0)\end{array}$ & $\begin{array}{c}20,0 \\
(0,6-89,0)\end{array}$ & $\begin{array}{c}58,0 \\
(29,0-82,0)\end{array}$ & $\begin{array}{c}21 \\
(5,0-51,0)\end{array}$ & $\begin{array}{c}21 \\
(5,0-51,0)\end{array}$ & 0 \\
\hline $\begin{array}{l}\text { Limpieza } \\
\text { social } \\
(n=14)\end{array}$ & $30(13)$ & $\begin{array}{c}92,9 \\
(66,0-99,8)\end{array}$ & $\begin{array}{c}69,2 \\
(39,0-91,0)\end{array}$ & $\begin{array}{c}42,9 \\
(10,0-82,0)\end{array}$ & $\begin{array}{c}14,3 \\
(0,4-58,0)\end{array}$ & $\begin{array}{c}14,3 \\
(0,4-58,0)\end{array}$ & $\begin{array}{c}33,0 \\
(10,0-65,0)\end{array}$ & $\begin{array}{c}17 \\
(2,0-48,0)\end{array}$ & $\begin{array}{c}25 \\
(6,0-57,0)\end{array}$ & $\begin{array}{c}25 \\
(6,0-57,0)\end{array}$ \\
\hline $\begin{array}{l}\text { Intervención } \\
\text { legal }(n=4)\end{array}$ & $34(10)$ & 100,0 & $\begin{array}{c}66,7 \\
(9,0-99,0)\end{array}$ & $\begin{array}{c}66,7 \\
(9,0-99,0)\end{array}$ & $\begin{array}{c}50,0 \\
(1,0-99,0)\end{array}$ & $\begin{array}{c}67 \\
(9,0-99,0)\end{array}$ & $\begin{array}{c}33,0 \\
(1,0-91,0)\end{array}$ & 0 & $\begin{array}{c}67 \\
(9,0-99,0)\end{array}$ & 0 \\
\hline $\begin{array}{l}\text { Maltrato } \\
\text { conyugal } \\
(n=1)\end{array}$ & 31 & 100,0 & 100,0 & 100,0 & 0,0 & 100 & 100,0 & 0 & 0 & 0 \\
\hline $\begin{array}{l}\text { Desconocido } \\
(n=1.179)\end{array}$ & - & $\begin{array}{c}88,5 \\
(87,0-90,0)\end{array}$ & $\begin{array}{c}75,7 \\
(73,0-78,0)\end{array}$ & $\begin{array}{c}90,7 \\
(36,0-41,0)\end{array}$ & $\begin{array}{c}0,2 \\
(0,0-0,7)\end{array}$ & $\begin{array}{c}12,9 \\
(9,0-20,7)\end{array}$ & $\begin{array}{c}41,0 \\
(38,0-45,0)\end{array}$ & $\begin{array}{c}16 \\
(14,0-19,0)\end{array}$ & $\begin{array}{c}42 \\
(39,0-45,0)\end{array}$ & $\begin{array}{c}1 \\
(0,3-1,6)\end{array}$ \\
\hline
\end{tabular}

* Promedio (desviación estándar).

** Un sólo muerto en el hecho. 
IC95\%: 8,0\%-11,0\%), Manrique (9,0\%; IC95\%: 7,0\%-11,0\%) y Aranjuez (8,9\%; IC95\%: 7,0\%$11,0 \%)$ del oriente y Doce de Octubre $(8,9 \%$; IC95\%: 7,0\%-11,0\%) del occidente.

Los barrios Popular (3,5\%; IC95\%: 2,5\%-5,0\%), Castilla (2,2\%; IC95\%: 1,5\%-3,0\%), Trinidad (1,7\%; IC95\%: 1,0\%-3,0\%), Kennedy (1,6\%; IC95\%: 0,9\%-2,6\%), Santo Domingo Savio \#1 (1,6\%; IC95\%: 0,09\%-2,6\%), La Esperanza (1,5\%; IC95\%: 0,9\%-2,4\%), Moravia (1,5\%; IC95\%: 0,9\%-2,4\%), San Bernardo (1,4\%; IC95\%: 0,8\%-2,3\%), Caicedo (1,3\%; IC95\%: 0,7\%-2,2\%) y Doce de Octubre \#1 (1,3\%; IC95\%: 0,7\%-2,2\%) tuvieron los porcentajes más altos de muertos residentes en ellos. Excepto Trinidad (Guayabal), San Bernardo (Belén) y Caicedo (Buenos Aires), los demás están ubicados en el norte de Medellín.

En las zonas Nor-Oriental y Centro-Oriental hubo el 57,4\% (IC95\%: 52,7\%-62,5\%) de los homicidios, mientras que en El Poblado sólo el 1,9\% (IC95\%: 1,2\%-2,8\%). La Candelaria, ubicada en el centro de la ciudad, fue en la comuna donde estuvo el mayor porcentaje de homicidios (16,2\%; IC95\%: 14,0\%-18,0\%). Los barrios donde ocurrieron más muertes fueron: Guayaquil (3,6\%; IC95\%: 2,7\%-4,8\%), Estación Villa (2,3\%; IC95\%: 1,6\%-3,3\%) y La Candelaria (1,9\%; IC95\%: 1,3\%-2,8\%) de la zona Centro-Oriental; Popular (2,2\%; IC95\%: 1,3\%-2,8\%), Moravia (1,8\%; IC95\%: 1,2\%-2,7\%), La Rosa (1,4\%; IC95\%: 0,8\%$2,2 \%)$, Granizal (1,2\%; IC95\%: 0,7\%-2,0\%) y Castilla (1,8\%; IC95\%: 1,2\%-2,7\%) de la zona Nor-Oriental; Doce de Octubre \#1 (1,6\%; IC95\%: $1,0 \%-2,6 \%)$ y Doce de Octubre \#2 (1,2\%; IC95\%: $0,7 \%-2,0 \%)$ de la Nor-Occidental; y Trinidad (1,4\%; IC95\%: 0,9\%-2,3\%)) en el Sur-Occidente.

El riesgo de morir porque una persona residiera en un barrio dado (ordenado de mayor a menor), que se estableció por el Índice $\mathrm{H} / \mathrm{P}$ por barrio de residencia, fue 1,4 veces mayor para quienes residían en Trinidad (Guayabal), que fue el barrio de más riesgo, que para los que lo hacían en Santa Lucía-Las Estancia (Villa Hermosa) que lo seguían en orden de riesgo, 1,9 veces que los del barrio Oriente (Manrique), 2,9 veces los del Barrio Colombia (El Poblado) y 3,1 veces que para Versalles \#2 (Manrique). El mayor riesgo en Trinidad es cerca de 25 veces más alto que en barrios que tienen un Índice de 1,0, tales como San José La Cima \#2 (Manrique), La Esperanza (Doce de Octubre) y Santa Margarita en Robledo (datos no presentados). La Figura 2 muestra los riesgos de homicidios por barrio de residencia (Índice H/P Barrio Residencia) categorizados en tres niveles ascendentes, Bajo, Medio y Alto.

En la Figura 3 están los riesgos de morir por residir en una comuna (Índice H/P Comuna de
Residencia) y el riesgo de morir por estar en una comuna (Razón H/P Comuna del Hecho). En el cuadrante superior derecho se ubican las comunas con los riesgos más altos y en el inferior izquierdo las comunas con los riesgos más bajos.

El riesgo de morir por homicidio en Medellín, según el estrato socioeconómico de residencia de las personas (Índice H/P Estrato de Residencia) fue menor de 1,0 para el estrato Bajo-bajo $(0,84)$, y tuvo una tendencia descendente a medida que aumenta: 1,19 en el estrato Bajo, 1,12 en el Medio-bajo, 0,98 para el Mediomedio, 0,26 para el Medio-alto y 0,13 en el Alto.

$\mathrm{Al}$ comparar estos Índices de los estratos se encuentra que, excepto para el 1, un estrato inferior tiene un riesgo mayor que cada estrato superior: el estrato 2 tiene 1,1 veces mayor riesgo que el estrato $3,1,2$ veces mayor que el \# 4, 4,6 veces mayor que el \# 5 y 9,2 veces el riesgo del \# 6.

Si se relaciona el barrio en el cual vivía el muerto con el barrio en el que lo asesinaron, se encontró que no se conocía el lugar de residencia en 361 casos (25,9\%; IC95\%: 24,0\%-28,0\%); los 1.033 hechos restantes ocurrieron en el mismo barrio o en uno cercano en el 56,7\% (IC95\%: 52,0\%-62,0\%), en un barrio alejado en el $42,4 \%$ (IC95\%: $39,0 \%-46,0 \%$ ) y los otros 10 habitaban en la calle.

El barrio del homicidio relacionado con el barrio de residencia no fue diferente según la edad, el sexo, la alcoholemia, ni el año, y son similares a las mostradas en la Tabla 2.

\section{Discusión}

Esta investigación presenta una visión panorámica de la muerte violenta en Medellín, Colombia, entre 1990 y 2002, período en el que se registraron las mayores tasas de homicidios de toda su historia y de la historia del país 14 y sobre la cual no existen estudios que comprendan todos esos años. Con ello se busca identificar regularidades, continuidades y especificidades, y responder a la falta de sistemas de información que proporcionen un conocimiento básico del comportamiento de este evento.

La mayoría de los trabajos disponibles sobre violencia homicida en Latinoamérica hacen alusión a la precariedad de la información con que se cuenta. En Colombia se estima en cerca del 18,0\% el subregistro en el total de defunciones (Rubio M. Comunicación personal; 1998). Sin embargo, el homicidio ha sido catalogado como el tipo de violencia para el cual las estadísticas son más confiables, sobretodo 
en las áreas urbanas, donde las distintas agencias gubernamentales se interesan en registrarlos y se esfuerzan en no ocultarlo. En nuestro país gozan de especial prestigio los datos aportados por el INMLyCF a partir de las necropsias realizadas por esta institución. Esta investigación tuvo al INMLyCF como fuente de los datos, lo que permite atribuirles una buena confiabilidad a los datos presentados; no sin advertir que el ocultamiento de los homicidios es una señal de la degradación de las confrontaciones y de la presencia de grupos con alto nivel de organización como efecto del poder económico y logístico a los cuales no ha sido ajena la ciudad de Medellín durante el período.

Los hallazgos muestran que la situación de los homicidios en Medellín no ha cambiado desde la década de los años ochenta 12. Su comportamiento se mantiene persistentemente elevado, aunque haya descendido a la mitad entre comienzos y finales de los noventa y a pesar de que haya un reconocimiento por las autoridades estatales y por la sociedad civil de que ellos son el principal problema social y de salud pública de la ciudad y de que el gobierno municipal haya hecho un empréstito con el Banco Interamericano de Desarrollo por 15 millones de dólares para enfrentarla 15. Las acciones del Estado han sido discontinuas y de alcances limitados, por la interrupción de las políticas entre los alcaldes y porque no se han dirigido a los centros neurálgicos de la violencia homicida, es decir, a las condiciones sociales que la posibilitan y la mantienen y porque, por el contrario, se han centrado en enfrentar la generada por el terrorismo y por la confrontación bélica entre él, los paramilitares y la guerrilla, que por su espectacularidad y barbarie centran la atención por la seguridad de la población.

Un estudio sobre mortalidad registrada en Medellín 16, indica que entre 1987 y 1996, las tasas de agresión con arma de fuego y explosivos ocuparon la primera posición y representaron el $61,0 \%$ de la mortalidad por todas las causas. Al centrar la observación en los homicidios, como ocurrió en esta investigación, se encuentra que cerca de 9 de cada 10 homicidios se realizaron con armas de fuego, en todos los años de estudio, lo que permite suponer un importante nivel de organización de la empresa homicida y refleja los niveles de armamentismo ilegal que favorecen la situación de violencia.

Los más afectados por los homicidios siguen siendo los hombres jóvenes, menores de 35 años, residentes en los barrios pobres de la ciudad, de estratos socioeconómicos bajos, donde predomina el desempleo o el empleo
Figura 2

Distribución del riesgo de muerte por homicidio según barrio de residencia. Medellín, Colombia, 1990/2002.

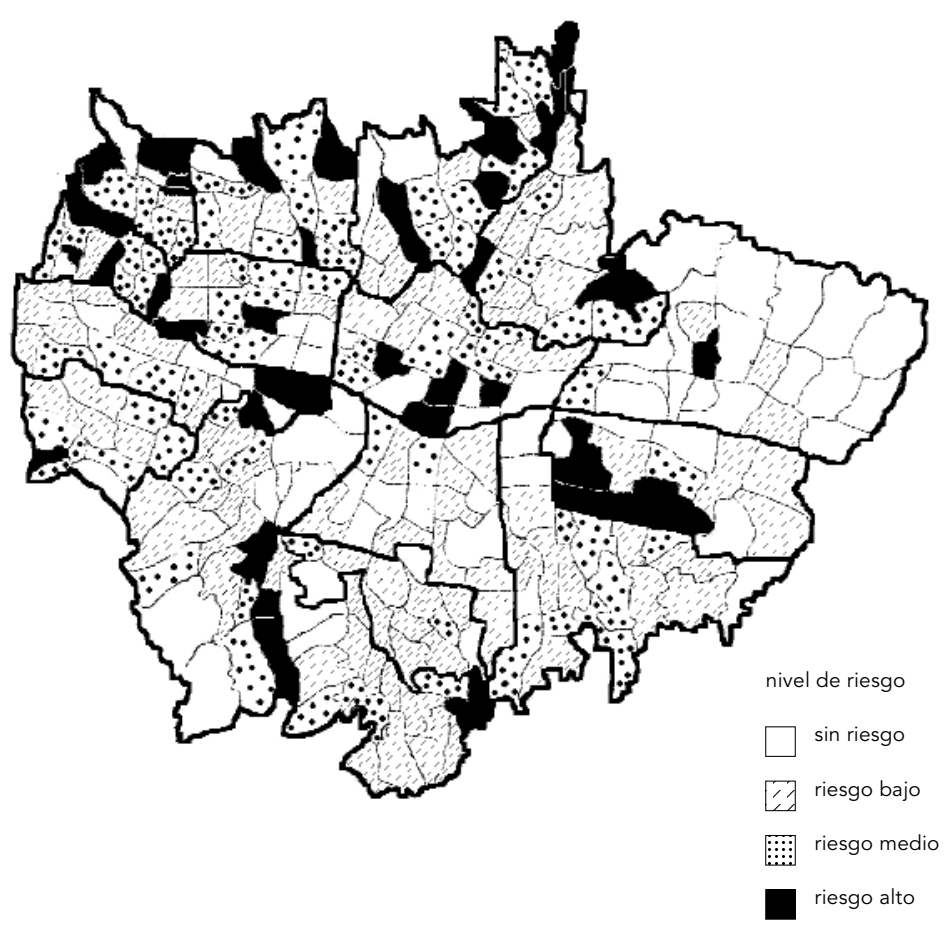

precario asociado a bajo nivel educativo y en donde la presencia social del Estado, con políticas sociales, es insuficiente o inexistente. De acuerdo con otros investigadores 17 , a partir de estos hallazgos, antes que estigmatizar los grupos y los barrios, lo que se requiere es leer los resultados como efectos de dinámicas sociales, donde la inequidad configura escenarios como referentes genealógicos de las vivencias de las comunidades, que almacenan, construyen y distribuyen capital social en y por medio de acciones colectivas y de acumulación de necesidades y de ausencias de Estado.

Otra característica que ha permanecido en la violencia en la ciudad es que se ejerce sobre sujetos individuales, donde las muertes múltiples o las matanzas son escasas y con asesinatos que no dejan heridos, aunque en una gran mayoría de casos se usen armas de fuego. A pesar de que los presuntos móviles de los homicidios se consignan en las Actas de levantamiento del cadáver, tan sólo en el $15,4 \%$, y de las limitaciones derivadas de la manera en que muchos casos se obtiene la información (por medio de testigos o con las personas que están 


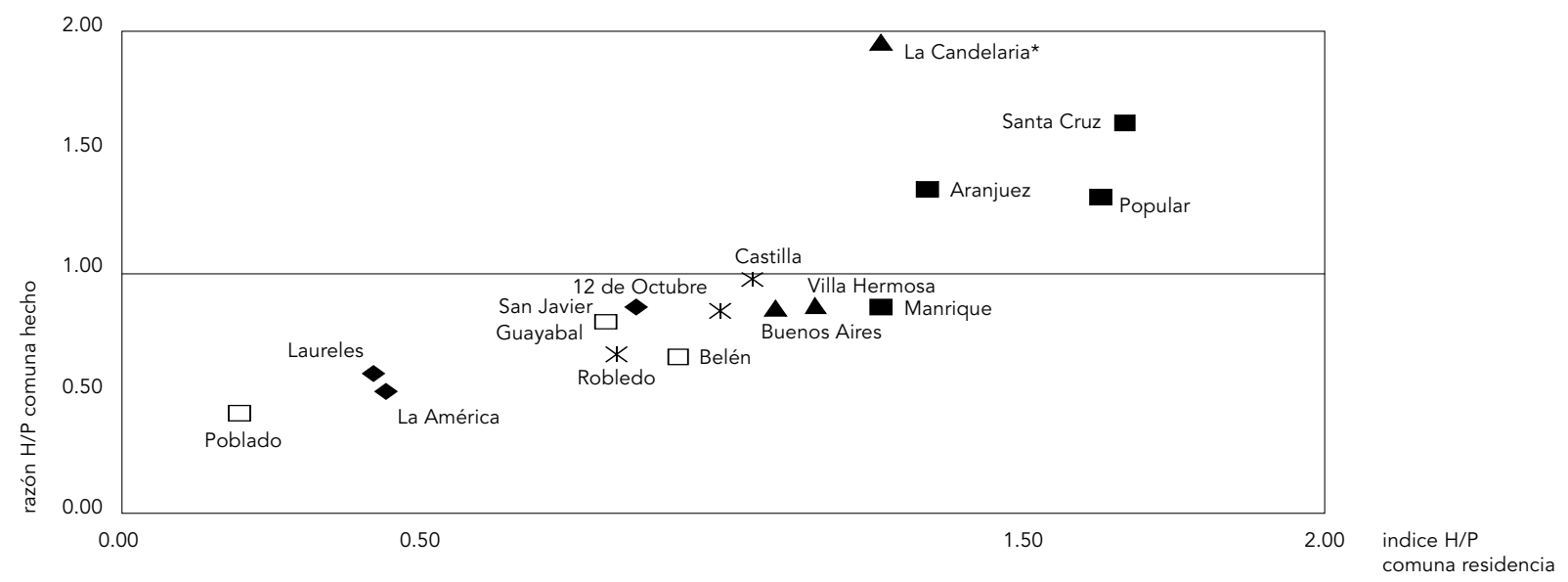

Índice lugar de residencia = \% homicidios de residentes en la comuna / \% población de la comuna.

Razón lugar del hecho = \% homicidios ocurridos en la comuna / \% población de la comuna.

* Razón $=4.18$

cerca del occiso en el momento de la diligencia), por lo descriptivo de la clasificación definida por el Instituto de Medicina Legal o por el interés predominantemente legal de su recolección 18, ellos muestran que en los homicidios predominan motivaciones personales, mediadas o no por la racionalidad, tales como los ajustes de cuentas, los atracos y las riñas.

El carácter descriptivo, retrospectivo y con base en fuentes secundarias de esta investigación, la limita a la calidad de las fuentes de información existentes y no permite establecer relaciones explicativas o de causalidad de los hallazgos. El alcohol ha encontrado con frecuencia en las personas asesinadas, en cifras tan elevadas como en Carolina del Norte y en Oklahoma, Estados Unidos, donde el $42,0 \%$ y el $52,0 \%$ estaban ebrios 19,20; aunque los positivos fueron menos (el 31,5\%) según un metanálisis que incluyó todos los estudios realizados en Estados Unidos 21. Las cifras encontradas en nuestro estudio fueron del $24,1 \%$, similares a las de Cali, Colombia, con el 18,0\% 11 .

La Comisión Económica para América Latina 22 compara la tasa de homicidio de Latinoamérica, que es de 20 por cien mil habitantes, y observa como punto extremo la situación que presenta Colombia, y dentro de ella Medellín, que se aleja completamente del promedio general y de ciudades como Bogotá, con cuatro veces la población de la ciudad. Este fenómeno se atribuye a la ruptura de las reglas de juego de la convivencia en los centros urbanos, donde los ciudadanos se ven amenazados y replegados a la individualidad, con escasos procesos de socialización ocasionada por la aparición de grupos al margen del estado como las guerrillas y paramilitares que se apoderan de los espacios más populares, en los cuales, por una parte se reflejan las grandes inequidades de las ciudades y, por otra, son el asentamiento de desplazados que encuentran en la ciudad el espacio para la supervivencia.

En estudios realizados en el país 14 (Rubio P. Comunicación personal; 1998) se encuentra en el aspecto económico una explicación a nuestra violencia homicida; más precisamente, la distribución arbitraria y desigual de la riqueza como generadora de inequidades y de marginalidad en grupos donde la violencia encuentra el espacio propicio para su desarrollo. La situación política, con la precaria formación y función del Estado que por una parte deja abiertas las posibilidades de toma y abuso del poder por grupos ilegales y por otro lado genera procesos de exclusión con fenómenos como la corrupción y el clientelismo.

Otra posible explicación es el proceso de urbanización de las ciudades que se ha dado sin una modernización social y económica, donde es característico un proceso de hibridación que destruye las identidades étnicas, con predominio de mezclas en todas las áreas, tanto culturales, sociales y políticas con primacía 
del sentido de lo efímero y del consumo; y en último término, la dinámica más compleja de lo urbano tiene que ver con la des-territorialización, categoría que incluye situaciones de desarraigo, de salidas, de llegadas, de emigraciones e inmigraciones de los pueblos a las ciudades, de las ciudades pequeñas a las ciudades grandes y de estas a la capital del país. Dicha categoría se refiere también al surgimiento de culturas sin memoria territorial, y aunque parezca paradójico significa des-urbanización en el sentido en que las ciudades son menos utilizables, más fragmentadas y dispersas. Estas explicaciones permiten conceptuar y tipificar diferentes violencias que provienen de intencionalidades distintas y se desarrollan tanto espacios públicos como privados 23 .

Si se tienen en consideración las propuestas explicativas planteadas y se relacionan con los resultados de este estudio, adquieren relevancia hallazgos como la cercanía entre el lugar de residencia del fallecido y el de ocurrencia de los hechos, así como lo encontrado con respecto al riesgo de morir según el lugar de residencia, de ocurrencia del hecho y del estrato socioeconómico. Lo anterior abre posibilidades de reflexión sobre las dinámicas geográficas y territoriales en las cuales se fragua la violencia homicida, más allá de las condiciones de pobreza.

Así, es posible suponer la existencia de jóvenes involucrados en sociabilidades marginales, constituidos en grupos que potencian su accionar fuera de la legalidad, como un denominador común en la ciudad de Medellín, en las zonas de frontera desde mediados de los años setenta y que se prolonga hasta la época actual. Jóvenes de vida corta que satisfacen en ese entorno sus necesidades de ser adultos, de tener dinero, de tener un espacio social de reconocimiento, de proveerse de las oportunidades que su familia no les puede brindar, incluso de ser proveedores económicos de una familia de condiciones precarias. No se trata de un fenómeno exclusivamente juvenil, dado que es un accionar que coexiste con las demás expresiones de los colectivos en los que se desenvuelven, ni tampoco es una expresión característicamente mortífera, puesto que coincide con las expresiones vitales de las que se ocupan las comunidades en las que viven que no los consideran como extraños a ellas y que les asignan funciones de suplencia de necesidades colectivas como es la de ser fuente de seguridad para la comunidad y agentes de justicia.

Sobre este sustrato se asientan fenómenos que hablan de propósitos ajenos a las comunidades que tienen un carácter más coyuntural, como los derivados del narcotráfico, la guerrilla, las autodefensas y otros intereses menos visibles. Estos últimos actores tienen como característica el ser fuente de poder, de dinero, de mayores capacidades logísticas y de generar lazos de pertenencia y de fidelidades a las que los jóvenes se someten sin reservas mientras dura su hegemonía en el territorio que habita, pero que los puede cambiar, si las circunstancias se modifican. Desde esta perspectiva podemos configurar un personaje multifacético: un joven, recluido en una zona de frontera, sin posibilidades de acceder a las oportunidades que genera la ciudad formal, que en ocasiones autoconstruye sus oportunidades, pero que predominantemente está dispuesto a las que le vienen de fuera. Una vez ejerce como testigo, en otra de homicida, en otra de víctima y en otra surte los espacios de la cárcel.

Este enfoque intenta prevenir de los estereotipos que ubican a los homicidas de un lado y a las víctimas de otro, que estigmatizan a zonas de la ciudad como peligrosas y fuentes de violencia, para pensar que en esas zonas habitan los que van a morir y que en los mismos espacios, en los que tiene lugar la violencia y la criminalidad se fragua la vida. Este planteamiento permite responder al propósito de avanzar más allá de contar los homicidios, hacia generar construcciones que tengan potencial explicativo, que abran alternativas de solución y que recuperen la palabra de las víctimas ya producidas y de las potenciales. 


\section{Resumen}

En Medellín, Colombia, el homicidio es la primera causa de morbimortalidad desde hace 20 años y sus tasas de mortalidad superan las de grandes capitales de América Latina. Se describen los actores, las circunstancias y los móviles de los homicidios en Medellín entre 1990 y 2002. De los 55.365 homicidios del período se estudiaron aleatoriamente 1.394, el 93,6\% (IC95\%: 92,2\%-94,8\%) eran hombres, el 77,0\% (IC95\%: 75,0\%79,5\%) menores de 35 años, una cuarta parte estaban ebrios y en 9 de cada 10 fueron muertos con armas de fuego. Los principales móviles del homicidio fueron los ajustes de cuentas y los atracos. El 37,0\% (IC95\%: 34,0\%-41,0\%) de los muertos provenían de los estratos más bajos de la ciudad y el riesgo de homicidio fue mayor a medida que el estrato socioeconómico era menor. Las características de los asesinatos en Medellín han permanecido invariables desde la década de los ochenta, cuando se inició el período de violencia más alto en la historia de la ciudad. Los grupos más afectados son los hombres jóvenes que viven y mueren en los barrios de estratos socioeconómicos más bajos, en hechos individuales que no dejan heridos.

Violencia; Homicidio; Factores Socioeconómicos

\section{Colaboradores}

M. Cardona, H. I. García, C. A. Giraldo, M. V. López y C. M. Suárez son los responsables de la concepción y diseño de la investigación y de la redacción del artículo; M. Cardona, H. I. García, C. A. Giraldo, M. V. López, C. M. Suárez, D. C. Corcho y C. H. Posada participaron en la conducción, ejecución, recolección de los datos y análisis de la información; M. N. Flórez dirigió y supervisó la recolección de los datos y participó en el procesamiento y análisis de ellos; H. I. García es el responsable de la integridad del artículo y de su envío a Cadernos de Saúde Pública.

\section{Agradecimientos}

A los funcionarios del Instituto Nacional de Medicina Legal y Ciencias Forenses Regional Nor-Occidente por permitirnos acceder a sus archivos de necropsias.

\section{Referencias}

1. Franco S. Violencia y salud en Colombia. Rev Panam Salud Pública 1997; 1:93-103.

2. Alcaldía de Medellín. Plan de desarrollo 20012003. Medellín competitiva: hacia una revolución de la cultura ciudadana. Medellín: Alcaldía de Medellín; 2001.

3. Metrosalud. Diagnóstico de la situación de salud de Medellín 1984-1993. Medellín: Metrosalud, Municipio de Medellín; 1994.

4. Servicio Seccional de Salud de Antioquia/Metrosalud/Universidad de Antioquia/Hospital Universitario San Vicente de Paúl/Instituto de Medicina Legal. La violencia en Antioquia: una crisis que compromete a todos. Boletín Epidemiológico de Antioquia 1987; 12:5-37.

5. Metrosalud. Cronología de la atención en salud de Medellín 1986-1996. Medellín: Metrosalud, Municipio de Medellín; 1997.

6. Departamento Administrativo de Planeación, Municipio de Medellín. Anuario estadístico de Medellín. Medellín: Municipio de Medellín; 2002.

7. Jaramillo FJ, Turizo RA. Mortalidad violenta en Medellín durante el 2002. Boletín del Centro de Referencia Nacional sobre Violencia, Instituto Nacional de Medicina Legal y Ciencias Forenses 2003; 8:1-4.

8. Sader E. La violencia urbana en Brasil. El caso de Río de Janeiro. In: Concha A, Carrión F, Cobo G, editores. Ciudad y violencias en América Latina. Cali: Gestión Urbana; 1994. p. 257-64.

9. Kliksberg B. El crecimiento de la criminalidad en América Latina. Washington DC: Banco Interamericano de Desarrollo; 2002.

10. Llorente MV, Echandía C, Escobedo R, Rubio M. Violencia homicida y estructuras criminales en Bogotá. Análisis Político 2001; 44:17-38.

11. Concha-Eastman A, Espitia VE, Espinosa R, Guerrero R. La epidemiología de los homicidios en Cali, 1993-1998: seis años de un modelo poblacional. Rev Panam Salud Pública 2002; 12:230-9.

12. García HI, Vélez CH. Caracterización de la violencia homicida en la década de los ochenta: aproximación a la construcción de escenarios y campos de conflicto [Tesis de Maestría]. Medellín: Facultad Nacional de Salud Pública, Universidad de Antioquia; 1992.

13. Resolución 000414. Por la cual se definen los parámetros científicos y técnicos relacionados con el examen de embriaguez y alcoholemia. Santafé de Bogotá: Instituto Nacional de Medicina Legal y Ciencias Forenses; 2002.

14. Franco S. El quinto: no matar. Contextos explicativos de la violencia en Colombia. Santafé de Bogotá: Tercer Mundo Editores; 1999.

15. Municipio de Medellín/Banco Interamericano de Desarrollo. Convenio para el plan de paz y convivencia en Medellín. Medellín: Municipio de Medellín/Banco Interamericano de Desarrollo; 1998.

16. Grisales H, Agudelo HB, Londoño JL. Mortalidad por las cinco primeras causas y su modelación temporal, Medellín, 1987-1996. Revista Facultad Nacional de Salud Pública 1998; 16:27-50.

17. Daza A, Salazar G. Hacia una estrategia de reconstrucción cultural. In: Daza A, editor. Experien- 
cias de intervención en conflicto urbano. Medellín: Programa de Convivencia Ciudadana; 2001.

18. Franco S, Forero LJ. Salud y paz en un país en guerra: Colombia, año 2002. In: Franco S, editor. La salud pública hoy. Enfoques y dilemas contemporáneos en Salud Pública. Santafé de Bogotá: Universidad Nacional de Colombia; 2003. p. 555-83.

19. Rutledege R, Messick W. The association of trauma death and alcohol use in a rural state. J Trauma 1992; 33:737-42.

20. Goodman R, Istre G, Jordan F, Herndon J, Kelaghan J. Alcohol and fatal injuries in Oklahoma. J Stud Alcohol 1991; 52:156-61.

21. Smith G, Branas C, Miller T. Fatal nontraffic injuries involving alcohol: a metaanalysis. Ann Emerg Med 1999; 33:359-68.

22. Arriagada I, Godoy L. Seguridad ciudadana y violencia en América Latina: diagnóstico y políticas en los años noventa. Santiago de Chile: Naciones Unidas/Comisión Económica para América Latina y el Caribe; 1999.

23. Camacho A, Guzmán A. Colombia: ciudad y violencia. Santafé de Bogotá: Ediciones Foro Nacional; 1990.

Recibido el 11/May/2004

Versión final presentada el 10/Sep/2004

Aprobado el 19/Oct/2004 